\title{
Sellar and Suprasellar Tuberculoma
}

\author{
Manpreet Singh Banga ${ }^{1} \quad$ Kaushik Roy ${ }^{2}$ Sandeep B. V. ${ }^{1} \quad$ Sibaji Dasgupta ${ }^{1}$
}

1Department of Neurosurgery, Nil Ratan Sircar Medical College, Sealdah, Kolkata, West Bengal, India

${ }^{2}$ Department of Neurosurgery, Nil Ratan Sircar Medical College, Sealdah, Kolkata, West Bengal, India

Indian J Neurosurg 2018;7:39-42

\author{
Address for correspondence Manpreet Singh Banga, MS, \\ Department of Neurosurgery, Nil Ratan Sircar Medical College, 138, \\ AJC Bose Road, Sealdah, Kolkata 700014, West Bengal, India \\ (e-mail: dr.msbanga@gmail.com).
}

\begin{abstract}
Keywords

- pituitary

- tuberculosis

- antitubercular therapy

Tuberculosis is responsible for $20 \%$ of the intracranial space-occupying lesions in India, and tuberculomas of the sellar and suprasellar region comprise only $1 \%$ of all intracranial tuberculomas. The clinical and radiological features of these lesions mimic a typical pituitary adenoma. Surgery is not usually indicated, except for obtaining biopsies to confirm diagnosis, as these lesions tend to resolve with appropriate antitubercular therapy. There is no consensus regarding the type of antitubercular regimen and duration of the treatment as the experience with tuberculomas of pituitary is limited. We report a rare case of hypophyseal tuberculosis in a patient as a sequela of tuberculous meningitis, which is a rare complication of tubercular meningitis.
\end{abstract}

\section{Introduction}

Inflammatory pituitary lesions are extremely rare, with an annual incidence estimated to be one case in 10 million. ${ }^{1}$ Histologically, these can be granulomatous, lymphocytic, xanthomatous, and necrotizing hypophysitis. Isolated involvement of pituitary gland by tuberculosis is very rare. Tuberculosis is responsible for $20 \%$ of the intracranial space-occupying lesions in India, and tuberculomas of the sellar and suprasellar region comprise only $1 \%$ of all intracranial tuberculomas. ${ }^{2}$ The need to investigate it early during diagnostic evaluation is of a major concern since the condition is reversible with antitubercular therapy (ATT). We report a rare case of hypophyseal tuberculosis in a patient as a sequela of tuberculous meningitis (TBM).

\section{Case Report}

A 19-year-old nonalcoholic male referred to our outpatient department as a follow- up case of TBM and was on ATT since 6 months. He presented with headache and severe diminution of vision in the left eye along with field defects in the form of temporal hemianopia in the right eye. Physical examination was unremarkable. Visual assessment showed a visual acuity of 6/18 in the right eye and no perception of light in the left eye. Fundus examination showed temporal pallor in the right eye and early optic atrophic changes in the left eye. Automated perimetry showed absolute temporal field defect in the right eye. Magnetic resonance imaging (MRI) of the brain showed enhancing lesion in the sellar and suprasellar region reported as pituitary macroadenoma, but the shape was suggestive of a meningioma; however, the imaging characteristic was not corroborating with typical meningioma. The lesion showed conglomerating pattern in one image, and thus a suspicion of tubercular lesion with a background of TBM could not be ruled out ( - Fig. 1 ). Erythrocyte sedimentation rate (ESR) was raised $(112 \mathrm{~mm}$ after 1 hour). His hormone profile was normal, except serum prolactin, which was mildly elevated, $34.21 \mathrm{ng} / \mathrm{mL}$. Serological test for human immunodeficiency virus was negative. Biopsy was planned for the lesion for which the patient was unwilling. We intensified the ATT and added short-term steroid therapy as the radiological images was suspicious of tuberculous infection of the pituitary gland. Patient had symptomatic improvement in headache and his visual acuity improved to 6/6 in the right eye and 6/60 in the left eye after 3 months. A repeat automated perimetry after 3 months showed significant improvement with absolute
DOI https://doi.org/

10.1055/s-0037-1599787. ISSN 2277-954X.
Copyright @2018 Neurological

Surgeons' Society of India
License terms

() (1) $\ominus \circledast$ 


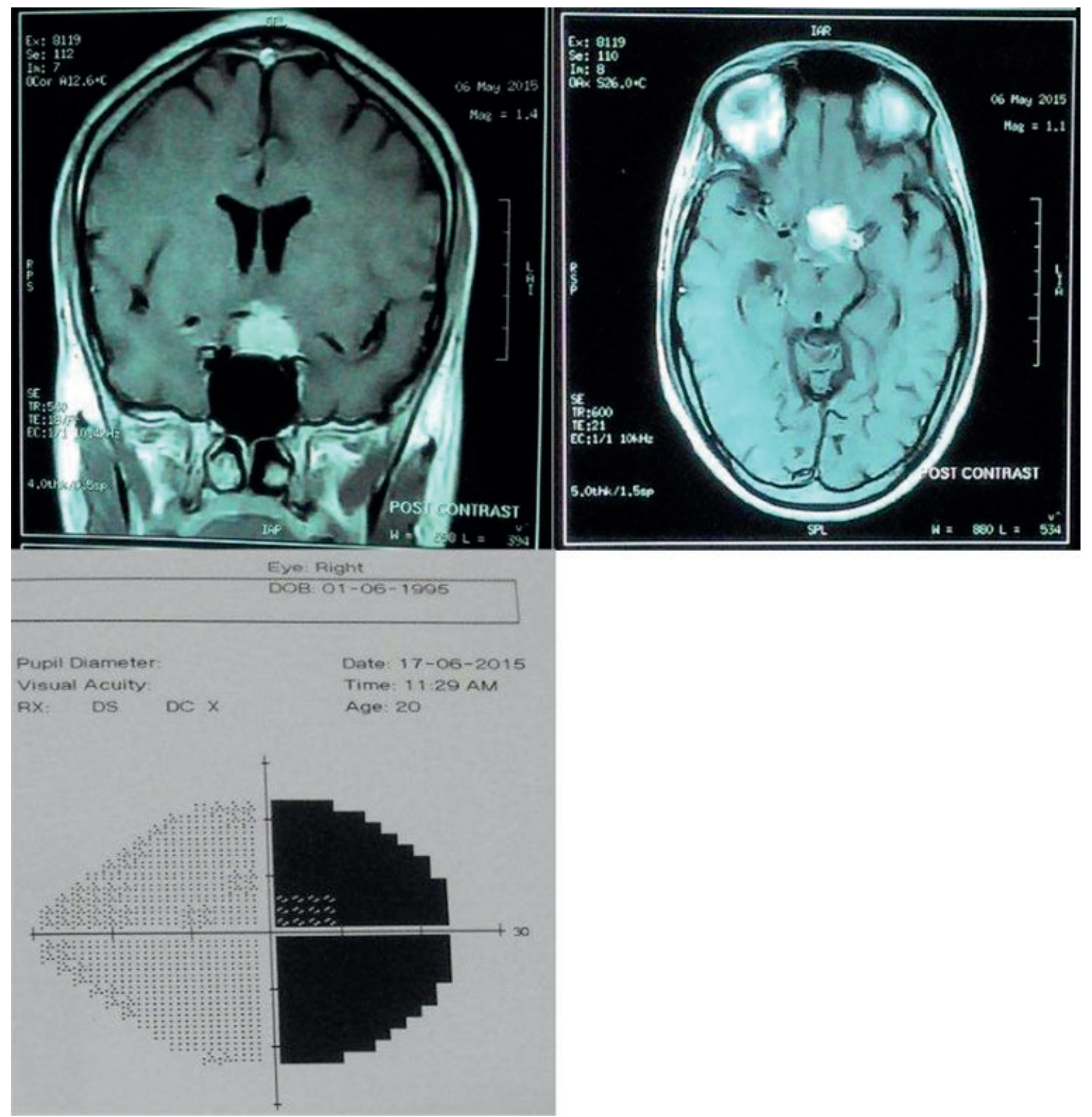

Fig. 1 Pre-treatment T1+ contrast coronal MRI showing enhancing sellar and suprasellar lesion. T1+contrast axial image showing conglomerating pattern of the lesion. Perimetry of right eye showing absolute temporal field defect.

upper temporal quadrant field defect in the right eye and absolute upper arcuate defect with lower temporal field defect in the left eye. Repeat MRI of the brain after a total of 12 months of ATT showed no mass lesion in the sellar and suprasellar region ( ). ESR normalized to $10 \mathrm{~mm}$ after 1 hour.

\section{Discussion}

Tuberculoma of the pituitary, although extremely infrequent, is a cause of secondary granulomatous hypophysitis, which is sometimes reported in developing countries. Although the first reported case of a sellar tuberculoma was from Britain in 1924, ${ }^{3}$ the literature on sellar tuberculomas, unsurprisingly, largely arises from the Indian subcontinent and other developing countries. Our patient presented with a history of headaches, visual field defect, and the background of treatment for TBM. Initial biochemistry was compatible with mild hyperprolactinemia, with normal levels of cortisol, gonadotrophins, thyroid profile, and growth hormone. Sharma et al, ${ }^{4}$ who have reported the largest series on intrasellar tuberculoma so far (18 cases), have described a $100 \%$ incidence of headaches and $38 \%$ incidence of endocrine disturbances including the amenorrhea-galactorrhea syndrome. A concomitant history of systemic tuberculoma is not always present. ${ }^{5}$ Radiologically, the tumor is usually sellar and suprasellar, being isointense on T1-weighted images and hyperintense on T2-weighted sequences. ${ }^{4}$ Pituitary stalk thickening is often noted on MR scans. ${ }^{6}$ The clinical and 


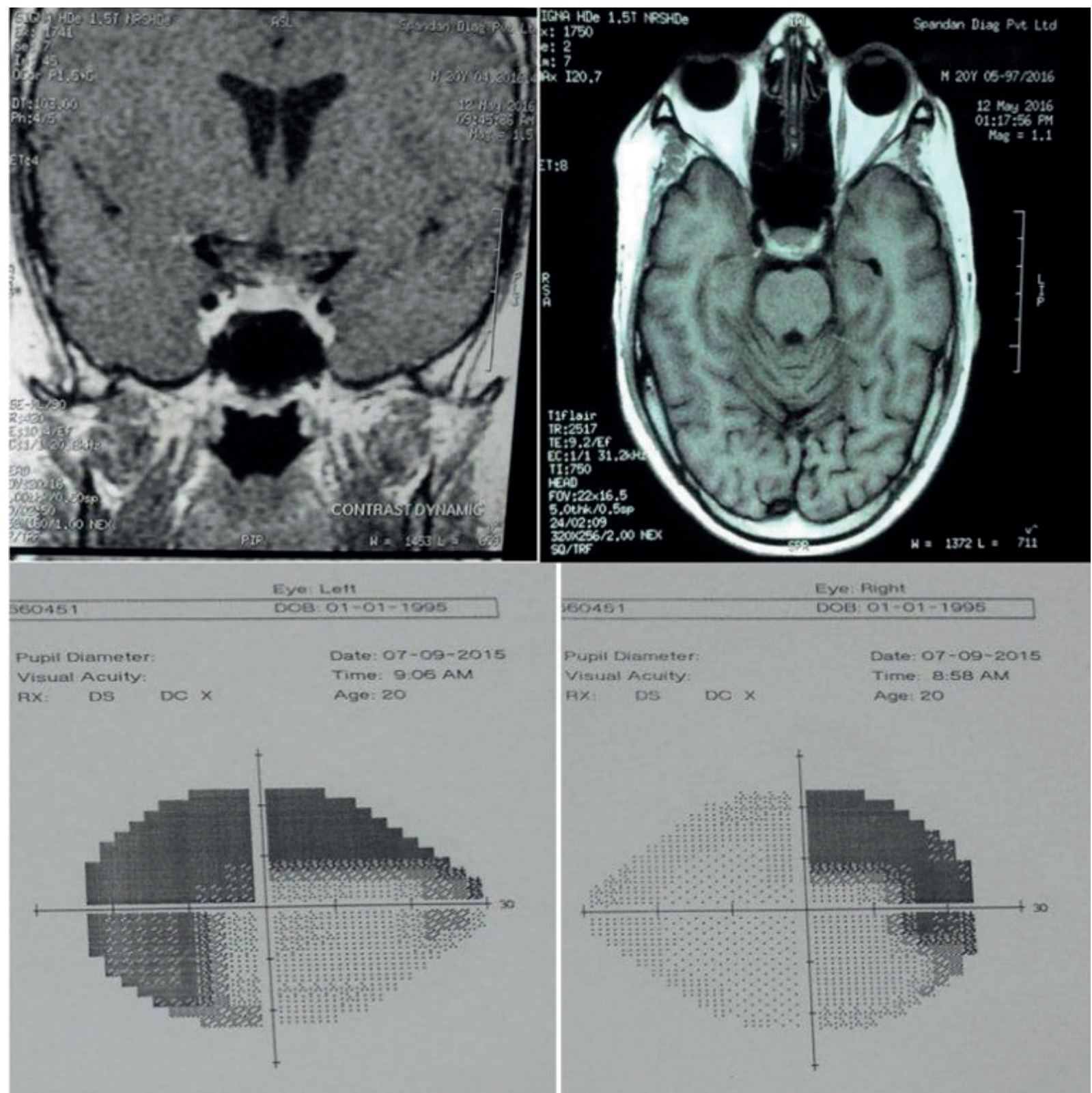

Fig. 2 MRI coronal and axial after 12 months ATT showing no mass lesion in sellar and suprasellar region. Repeat perimetry after 3 months of treatment with ATT showed absolute upper temporal quadrant field defect in right eye and absolute upper arcuate defect with lower temporal field defect in the left eye.

radiological features of these lesions mimic a typical pituitary adenoma. Surgery is not usually indicated, except for obtaining biopsies to confirm diagnosis, as these lesions tend to resolve with appropriate ATT. ${ }^{7}$ ATT is mandatory in all cases of pituitary tuberculosis. There is no consensus regarding the type of antitubercular regimen and duration of the treatment as the experience with tuberculomas of pituitary is limited. In our case, we had almost complete resolution of the lesion by 12 months. There are reports of marked resolution of the size of pituitary tuberculoma with ATT, which was started on the basis of clinical suspicion alone. ${ }^{7}$ There are reports of normalization of prolactin and improvement of diabetes insipidus on ATT without any specific treatment. ${ }^{8}$ Our case is remarkable as it points out to a very rare complication of TBM and has been reported rarely in the literature. It has been reported earlier by Mageshkumar et al. ${ }^{9}$

In conclusion, although diagnosis of sellar tuberculomas is difficult on clinical and radiological examinations, pituitary tuberculomas should be considered in the differential diagnosis of suprasellar masses, especially in developing countries. It is advisable to have tissue diagnosis before starting any specific treatment; however, antituberculous drugs can be started even on suspicion of pituitary TB. Long-term chemotherapy with antituberculous drugs usually results in a good outcome. Since it is not clear whether patients require lifelong replacement of deficient hormones, periodic follow-up is necessary. 


\section{References}

1 Sautner D, Saeger W, Lüdecke DK, Jansen V, Puchner MJ. Hypophysitis in surgical and autoptical specimens. Acta Neuropathol 1995;90 (06):637-644

2 Mascarenhas JV, Vageesh Ayyar S. Tuberculosis of the sella masquerading as pituitary adenoma. J Clin Sci Res 2013;2:42-44

3 Letchworth TW. Tuberculoma of the pituitary body. BMJ 1924;1(3313): 1127

4 Sharma MC, Arora R, Mahapatra AK, Sarat-Chandra P, Gaikwad SB, Sarkar C. Intrasellar tuberculoma-an enigmatic pituitary infection: a series of 18 cases. Clin Neurol Neurosurg 2000;102(02):72-77

5 Majumdar K, Barnard M, Ramachandra S, Berovic M, Powell M. Tuberculosis in the pituitary fossa: a common pathology in an uncommon site. Endocrinol Diabetes Metab Case Rep 2014;2014:140091

6 Domingues FS, de Souza JM, Chagas H, Chimelli L, Vaisman M. Pituitary tuberculoma: an unusual lesion of sellar region. Pituitary 2002;5 (03):149-153

7 Dutta P, Bhansali A, Singh P, Bhat MH. Suprasellar tubercular abscess presenting as panhypopituitarism: a common lesion in an uncommon site with a brief review of literature. Pituitary 2006;9 (01):73-77

8 Sunil K, Menon R, Goel N, et al. Pituitary tuberculosis. J Assoc Physicians India 2007;55:453-456

9 Mageshkumar S, Patil DV, Philo AJA, Madhavan K. Hypopituitarism as unusual sequelae to central nervous system tuberculosis. Indian J Endocrinol Metab 2011;(Suppl 3):S259-S262 